\title{
Surefire infusion system versus standard microcatheter use during holmium-166 radioembolization: study protocol for a randomized controlled trial
}

Andor F. van den Hoven ${ }^{*}$, Jip F. Prince, Rutger C. G. Bruijnen, Helena M. Verkooijen, Gerard C. Krijger, Marnix G. E. H. Lam and Maurice A. A. J. van den Bosch

\begin{abstract}
Background: An anti-reflux catheter (ARC) may increase the tumor absorbed dose during radioembolization (RE) by elimination of particle reflux and its effects on hemodynamics. Since the catheter is fixed in a centro-luminal position, it may also increase the predictive accuracy of a scout dose administration before treatment. The purpose of the SIM trial is to compare the effects of ARC use during RE with holmium-166 $\left({ }^{166} \mathrm{Ho}\right)$ microspheres in patients with colorectal liver metastases (CRLM), with the use of a standard end-hole microcatheter.

Methods/Design: A within-patient randomized controlled trial (RCT) will be conducted in 25 patients with unresectable chemorefractory liver-dominant CRLM. Study participants will undergo a ${ }^{166} \mathrm{Ho}$ scout dose procedure in the morning and a therapeutic procedure in the afternoon. The ARC will be randomly allocated to the left/right hepatic artery, and a standard microcatheter will be used in the contralateral artery. SPECT/CT imaging will be performed for quantitative analyses of the microsphere distribution directly after the scout and treatment procedure. Baseline and follow-up investigations include ${ }^{18} \mathrm{~F}-\mathrm{FDG}-\mathrm{PET}+$ liver $\mathrm{CT}$, clinical and laboratory examinations. The primary endpoint is the comparison of tumor to non-tumor ( $\mathrm{T} / \mathrm{N})$ activity ratio in both groups. Secondary endpoints include comparisons of mean absorbed dose in tumors and healthy liver tissue, infusion efficiency, the predictive value of ${ }^{166} \mathrm{Ho}$ scout dose for tumor response. In the entire cohort, a dose-response relationship, clinical toxicity, and overall survival will be assessed. The sample was determined for the expectation that the ARC will increase the T/N ratio by $25 \%$ (mean T/N ratio 2.0 vs. 1.6).
\end{abstract}

Discussion: The SIM trial is a within-patient RCT that will assess whether ${ }^{166} \mathrm{Ho}$ RE treatment can be optimized by using an ARC.

Trial registration: The SIM trial is registered at clinicaltrials.gov (NCT02208804). Registered on 31 July 2014.

\section{Background}

Hepatic radioembolization (RE) has evolved to a standardof-care therapy for patients with irresectable and chemorefractory colorectal cancer liver metastases (CRLM). During this treatment, radioactive microspheres are injected in the hepatic arteries to irradiate liver tumors from within, leading to local disease control [1]. However, treatment efficacy and predictability of the treatment effect can still be

\footnotetext{
* Correspondence: a.f.vandenhoven@umcutrecht.nl

Department of Radiology and Nuclear Medicine, University Medical Center

Utrecht, Heidelberglaan 100, 3584 CX Utrecht, The Netherlands
}

optimized, since the radiation dose to individual tumors is often inadequate and this cannot be predicted beforehand due to the lack of a reliable surrogate particle (scout dose) for routinely used yttrium-90 $\left({ }^{90} \mathrm{Y}\right)$ microspheres [2] .

Holmium-166 $\left({ }^{166} \mathrm{Ho}\right)$ microspheres have been developed and clinically validated as a new microsphere for RE [3]. Besides $\beta$-radiation, these microspheres also emit lowenergy $\gamma$-radiation and have paramagnetic characteristics, allowing for visualization and quantitative assessment of the microsphere distribution on single-photon emission computed tomography (SPECT) and magnetic resonance 
imaging (MRI) [4]. Furthermore, identical ${ }^{166}$ Ho microspheres can be administered as a scout dose to predict the distribution of the therapeutic microspheres $[3,5]$.

Another promising development is the availability of an anti-reflux catheter (ARC, Surefire Infusion System, Surefire Medical Inc., Westminster, CO, USA), aiming to increase the infusion efficiency and safety, by prevention of reflux [6-8]. The effects on hemodynamics induced by this catheter may improve the microsphere uptake ratio between tumorous and non-tumorous liver tissue $(\mathrm{T} /$ $\mathrm{N}$ ratio). The expanded catheter tip decreases blood pressure downstream of the tip, which is believed to lower the resistance that injected microspheres need to overcome in order to reach the tumor vasculature [9]. Furthermore, the catheter tip converts blood flow from a laminar into a turbulent pattern, which reduces the chance of missing a target branch by proper mixing of the microspheres in the vascular compartment. In addition, the catheter is fixed into a centro-luminal catheter position during infusion which may further increase the accuracy of the ${ }^{166} \mathrm{Ho}$ scout dose as a predictor for the therapeutic ${ }^{166}$ Ho microspheres distribution [2].

In this article, we describe the study protocol of a prospective, comparative, clinical trial with the aim to investigate whether the ARC can be used to improve tumor targeting as well as the predictability of the treatment effect during ${ }^{166}$ Ho RE.

\section{Methods}

\section{Hypothesis}

We hypothesize that the use of the ARC increases the posttreatment $\mathrm{T} / \mathrm{N}$ activity ratio and improves the predictive value of ${ }^{166} \mathrm{Ho}$ scout dose distribution, in comparison with the use of a standard microcatheter (SMC).

\section{Study design}

The SIM trial is a single-center, open-label, phase II, within-patient randomized controlled trial (RCT). As opposed to a conventional RCT design, patients act as their own controls; liver lobes (right versus left lobe) are the experimental units in this study. Patients with bilateral, irresectable, chemorefractory, liver-dominant CRLM will undergo two sequential procedures on the same day, during which a scout dose and a therapy dose of ${ }^{166} \mathrm{Ho}$ microspheres will be administered in the left and right hepatic artery. These separate injections can be regarded as two separate treatments of functionally independent liver lobes. The use of the ARC will be randomly allocated (1:1) to the left or right functional liver lobe and a standard microcatheter (SMC) will be used in the contralateral side. The same catheter type will be used on the same side during the scout and therapy procedures.

This trial will be conducted in accordance with the principles outlined in the Declaration of Helsinki and will follow the Consolidated Standards of Reporting Trials (CONSORT) statement [10]. A Standard Protocol Items: Recommendations for Interventional Trials (SPIRIT) checklist is provided in Additional file 1 [11].

\section{Study population}

All patients with irresectable, chemorefractory, and liverdominant CRLM are eligible for participation in this trial if they meet the following inclusion criteria: histopathologically confirmed diagnosis of adenocarcinoma of the colon or rectum, hepatic metastases with measurable morphological appearance $(\geq 1 \mathrm{~cm})$ on cross-sectional imaging located in both the right and left hepatic arterial perfusion territory, irresectable and liver-dominant disease (i.e., pathological locoregional lymph nodes and up to five lung lesions < $1 \mathrm{~cm}$ are accepted), progressive disease after standard second-line systemic treatment or no further systemic treatment options due to severe side effects or unwillingness of the patient, age $\geq 18$ years. Adequate follow-up must be logistically feasible and written informed consent must be obtained before enrollment in the study.

Exclusion criteria are: World Health Organization (WHO) performance score $>2$, inadequate bone marrow function (hemoglobin $<6.0 \mathrm{mmol} / \mathrm{l}$, leukocyte count $<$ $3.0 \times 10^{9} / \mathrm{l}$, platelet count $\left.<75 \times 10^{9} / \mathrm{l}\right)$, inadequate liver function (bilirubin $>35 \mu \mathrm{mol} / \mathrm{l}$, aspartate aminotransferase/ alanine aminotransferase $>5 \times$ upper limit of normal) or inadequate renal function (creatinine $>1.5 \times$ upper limit of normal), prior hemihepatectomy, compromised biliary system (biliary stent or hepaticojejunostomy), Child-Pugh score $\mathrm{B} 7$ or worse, active hepatitis $\mathrm{B}$ or $\mathrm{C}$, main portal vein thrombosis or previous portal vein embolization, severe celiac axis stenosis, unsuitable hepatic arterial anatomy, treatment with systemic chemotherapy within 4 weeks prior to RE, previous participation in a study classified as class III by a radiation safety committee, bleeding diathesis, pregnancy or breast feeding, or any condition that prevents safe treatment with RE.

\section{Investigations and interventions}

The flowchart in Fig. 1 shows how the investigations and interventions in the SIM trial compare to standard radioembolization practice with ${ }^{90} \mathrm{Y}$ microspheres.

\section{Baseline investigations}

Patients will first undergo pretreatment investigations as part of our routine RE workup: whole-body ${ }^{18} \mathrm{~F}$-fluorodeoxyglucose positron emission tomography/computed tomography $\left({ }^{18} \mathrm{~F}\right.$-FDG-PET/CT) to rule out extensive extrahepatic metastases and calculate metabolic tumor burden; dual-phase (arterial + portal phase) liver CT to rule out other contraindications for $\mathrm{RE}$, localize the liver tumors, and assess the hepatic arterial anatomy; laboratory 

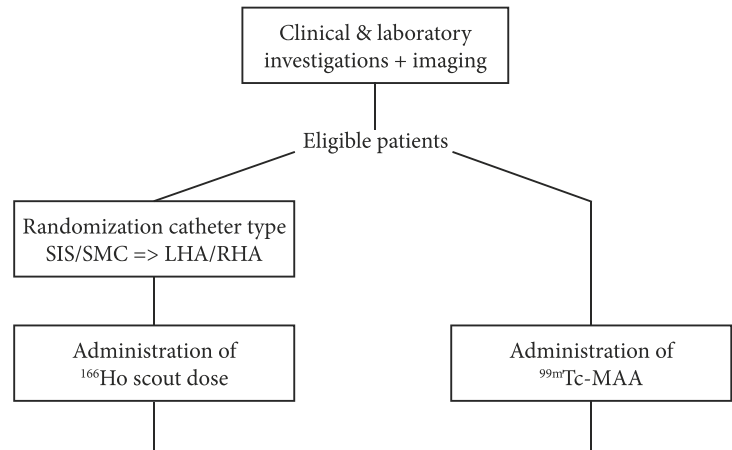
${ }^{99 \mathrm{~m}} \mathrm{Tc}-\mathrm{MAA}$



Fig. 1 Flowchart of the investigations and interventions in the SIM trial. The study procedures are compared to standard radioembolization practice with yttrium-90 $\left({ }^{90} \mathrm{Y}\right)$ microspheres. Note that in the SIM trial, the same particle is used during the scout and therapy procedure, and patients receive all procedures on the same day

investigations and a physical examination to assess vital functions and general condition of the patient.

\section{Treatment planning}

Eligible patients will be asked to provide informed consent to participate in the study. Next, the interventional radiologist and nuclear medicine physician will make an individualized treatment plan (including identification of the target vessels for selective infusion, identification of extrahepatic branches, and determining the need for coil embolization), based on the anatomy of the hepatic arterial vasculature visualized on pretreatment CT images. In all procedures, two selective injection positions (in the left and right hepatic artery) will be used to treat the whole liver on the same day. In patients with a variant hepatic arterial configuration, intrahepatic arterial branches (for example, the segment 4 artery) may be coil embolized to induce redistribution of blood flow through intrahepatic collaterals, and enable the use of two selective injection positions.
The total amount of ${ }^{166}$ Ho activity $\left(\mathrm{A}_{\mathrm{Ho166}}\right)$ required to deliver a whole liver absorbed dose of $60 \mathrm{~Gy}$ - the maximum tolerable radiation dose previously determined in a phase I dose-escalation study - is calculated using the formula:

$$
A_{H o 166}(M B q)=\frac{60(G y)}{15.87 \times 10^{-3}(J / M B q)} x \text { Liverweight }(K g)
$$

Liver weight (in $\mathrm{Kg}$ ) is determined by multiplying the volume of the liver (measurement based on $\mathrm{CT}$ ) with the density of liver tissue $\left(1.06 \mathrm{~g} / \mathrm{cm}^{3}\right)$. The scout procedure always consist of $250 \mathrm{MBq}$ in $60 \mathrm{mg}$ of microspheres (density $1.4 \mathrm{~g} / \mathrm{cm}^{3}$ ), this activity is subtracted from the activity given in the treatment procedure. The treatment activity for each perfusion territory is fractionated based on its liver volume. If coil embolization of an intrahepatic branch is planned, the volume of its perfusion territory is included in the side that is anticipated to take over the blood supply. 


\section{Scout and therapy procedures}

All patients will be admitted to hospital (University Medical Center Utrecht, The Netherlands) and will undergo a ${ }^{166} \mathrm{Ho}$ scout procedure the next morning, followed by the ${ }^{166} \mathrm{Ho}$ therapeutic procedure in the afternoon when the scout procedure was performed successfully.

During the ${ }^{166} \mathrm{Ho}$ scout procedure, digital subtraction angiography (DSA) and C-arm CT imaging will be performed to confirm adequacy of the intended injection position(s), and rule out potential extrahepatic shunting. Coil embolization of side branches will only be performed when inevitable to warrant safe treatment. Nitroglycerine $200 \mu \mathrm{g}$ is given per intra-arterial catheter to prevent vasospasm. Ultimately, a scout dose $(250 \mathrm{MBq})$ of ${ }^{166} \mathrm{Ho}$ microspheres will be administered through the anti-reflux catheter (ARC) and the SMC in the target vessels.

Next, a SPECT scan combined with a portal venous phase liver $\mathrm{CT}$ will be acquired and evaluated to rule out extrahepatic deposition of ${ }^{166} \mathrm{Ho}$ microspheres, assess the intrahepatic biodistribution, as well as the liver-to-lung shunt. In the absence of extrahepatic deposition and a significant liver-to-lung shunt (visible accumulation ${ }^{166} \mathrm{Ho}$ in the lung parenchyma), patients will undergo the therapeutic procedure in the afternoon. In the case of an unsuccessful scout procedure, the patient will be scheduled for a repeat procedure if possible.

During the therapeutic procedure, the calculated treatment activity of ${ }^{166} \mathrm{Ho}$ microspheres will be administered in the same position(s) as during the scout procedure. Patients are discharged the day after treatment. The posttreatment SPECT (combined with a dual-phase liver CT) is obtained approximately 5 days after treatment to reduce the influence of detector dead time.

\section{Follow-up investigations}

A telephonic consultation will be scheduled at 2 weeks after treatment to evaluate the patient's general condition, and ask for the occurrence of adverse events. One month after treatment, patients will undergo a physical examination and laboratory investigations for toxicity assessment. At 3month follow-up, this is complemented by a whole-body ${ }^{18}$ F-FDG-PET + dual-phase liver CT for tumor response assessment, after which the study follow-up is completed.

\section{Study objectives}

The primary objective is to assess the difference in posttreatment $\mathrm{T} / \mathrm{N}$ activity ratio on SPECT/CT between administration with the ARC and SMC.

Secondary objectives include comparison of the following endpoints between administrations with the ARC and SMC: mean tumor and healthy liver absorbed dose on SPECT/CT, percentage of calculated treatment activity administered (infusion efficiency), predictive value of the ${ }^{166} \mathrm{Ho}$ scout dose distribution, and posttreatment tumor response. Furthermore, the presence of a dose-response relation between tumor absorbed dose and posttreatment tumor response will be evaluated.

\section{Outcome assessment}

In every target vessels' perfusion territory, the absorbed dose on ${ }^{166} \mathrm{Ho}$-SPECT reconstructions will be determined for the metastases and the healthy liver tissue [4]. Consequently, a posttreatment $\mathrm{T} / \mathrm{N}$ activity ratio will be calculated for each perfusion territory by dividing the number of counts in tumorous and healthy liver tissue.

The predictive value of the scout dose will be assessed by comparison of the distribution of the scout dose with the treatment dose. The analysis will be based on tumor and healthy liver absorbed doses on SPECT/CT analysis.

Infusion efficiency is defined as the percentage of the prepared treatment activity that is infused. The ${ }^{166} \mathrm{Ho}$ activity that is not infused will be determined by measuring the administration system (vial, lines, and catheters) with a dose calibrator.

Response analysis will be performed in accordance with the Response Evaluation Criteria in Solid Tumors (RECIST) version 1.1 [12], for each functional liver lobe separately. Disease control rates (percentage of liver lobes with complete response, partial response or stable disease) will be determined by $\mathrm{CT}$ evaluation at 3 months posttreatment. Metabolic tumor response will be determined by assessing the change (relative to baseline) in total lesion glycolysis values on ${ }^{18}$ F-FDG-PET imaging. During the response assessment, observers will be blinded for the catheter type used.

The relationship between tumor absorbed dose on SPECT/CT and tumor response on both $\mathrm{CT}$ and ${ }^{18} \mathrm{~F}$ FDG-PET will be characterized on the level of perfusion territories.

Common Terminology Criteria for Adverse Events v 4.03 will be used to describe laboratory and clinical toxicity for the entire cohort, with specific attention for device-related adverse events per catheter type such as the occurrence of reflux, vasospasm, or arterial dissections.

The municipal administration will be consulted to assess the overall survival, measured from treatment onward.

\section{Statistical considerations Sample size calculation}

A subgroup analysis of the $\mathrm{T} / \mathrm{N}$ activity ratios in seven patients with CRLM, previously treated with ${ }^{166} \mathrm{Ho}$ RE using a SMC [3], showed a mean $\mathrm{T} / \mathrm{N}$ ratio of 1.6 (standard deviation 0.57). Sample size calculation, based on a two-sided paired $t$ test for the comparison of $\mathrm{T} / \mathrm{N}$ activity concentration ratios at an alpha level of 0.05 and power of 0.90 , showed that at least 23 patients need to be included to detect a difference of 0.4 in mean $\mathrm{T} / \mathrm{N}$ ratios (with an estimated standard deviation 0.57) in the 
ARC arm (estimated mean $\mathrm{T} / \mathrm{N}$ ratio 2.0) and SMC arm (estimated mean $\mathrm{T} / \mathrm{N}$ ratio 1.6 ). This corresponds to a $25 \%$ increase (considered clinically relevant) in favor of the ARC arm. A rounded number of 25 patients will therefore be treated in this trial. Sample size calculations were performed with the computer program 'PS: Power and Sample Size Calculation' version 3.0 for MacOsX.

\section{Randomization}

A computer-generated stratified permuted block rando mization with varying block sizes will be used. Difference in tumor burden $(<10 \%$ or $\geq 10 \%)$ between the two target volumes in the liver will be used as stratification factors to eliminate the potential influence of tumor burden on the comparison of $\mathrm{T} / \mathrm{N}$ activity ratios between catheter types.

\section{Statistical analysis}

A comparison of continuous outcome measures between $\mathrm{ARC}$ and SMC infusions, such as the mean $\mathrm{T} / \mathrm{N}$ activity concentration and mean decrease in total lesion glycolysis at 3 months posttreatment, will be performed by means of a paired $t$ test. Categorical outcome measures, such as infusion efficiency and disease control rates at 3 months posttreatment, will be compared with a McNemar's test. The value of the ${ }^{166} \mathrm{Ho}$ scout dose distribution will be assessed per catheter type. Correlation and agreement with the ${ }^{166}$ Ho therapy dose distribution will be evaluated with a linear regression analysis $\left(\mathrm{R}^{2}\right)$ and Bland-Altman analysis (limits of agreement), respectively. The doseresponse relationship will be evaluated by linear regression analysis. Survival analysis by the Kaplan-Meier method will be used to estimate the median overall survival time for the entire cohort. The primary analyses will be performed on an intention-to-treat (as randomized) basis.

A two-sided $p$ value $<0.05$ will be considered statistically significant.

\section{Study organization}

Data completeness and accuracy will be frequently checked by the Department of Radiology and Nuclear Medicine, University Medical Center Utrecht. An independent clinical research associate (Julius Clinical, Zeist, The Netherlands) will audit the trial conduct during 3-4 visits. Appointing a data safety monitoring board was not required for this trial. As per Dutch regulations, serious adverse events, serious adverse device effects, and protocol violations are recorded and reported to the institutional review board. There are no stop rules in this study. No interim analysis is planned.

\section{Discussion}

In patients with advanced colorectal cancer, liver metastases are the primary cause of morbidity and mortality.
Unfortunately, only a minority of patients is a candidate for surgical resection with curative intent. Patients with irresectable disease will first receive palliative systemic therapy. Though, despite major advances in systemic treatment, overall survival remains disappointing in this subgroup of patients $[13,14]$.

Current standard of care in the systemic treatment of metastatic colorectal cancer is based on cytotoxic fluoropyrimidines, oxaliplatin, and irinotecan, as well as targeted therapy with the vascular endothelial growth factor (VEGF)-targeted monoclonal antibody bevacizumab [15-19]. There is no clear preference for sequential exposure to these drugs during consecutive lines of treatment or upfront combination therapy [20, 21]. When disease progression or intolerable toxicity occurs during first-line treatment, patients will subsequently receive another regimen as second-line treatment, with the choice of the regimen depending on the first chemotherapeutic agents. In the Netherlands, the first-line regimen of choice is CAPOX-B (capecitabine, oxaliplatin, and bevacizumab). After initial treatment with six cycles of CAPOX-B, maintenance therapy with capecitabine and bevacizumab is given until disease progression [22]. Subsequently an irinotecan-based regimen, e.g., FOLFIRI (leucovorin, fluorouracil (5-FU), and irinotecan) or irinotecan monotherapy, is indicated as secondline therapy. Only patients with a KRAS wild-type tumor may benefit from additional (third-line) treatment with an epidermal growth factor receptor (EGFR)-targeted monoclonal antibody (panitumumab or cetuximab).

Liver-directed therapy such as RE is increasingly applied as an alternative to achieve local disease control. Currently, two types of yttrium-90 microspheres are used in worldwide clinical practice: resin (SIR-Spheres, SIRTeX, Lane Cove, Australia) and glass (TheraSphere; BTG, Ottawa, ON, Canada) yttrium-90 ( $\left.{ }^{90} \mathrm{Y}\right)$ microspheres. In salvage patients with colorectal liver metastases, who have no regular treatment options left and an average life expectancy of less than 6 months, median overall survival after RE treatment with ${ }^{90} \mathrm{Y}$ microspheres is around 12 months when given as monotherapy or in combination with chemotherapy [1]. Besides, treatment is generally well tolerated, with typical clinical toxicity being limited to mild symptoms of fatigue, abdominal pain, nausea, vomiting and/or fever during the first 2 weeks after treatment [23].

Despite these benefits of RE, there is still room for improvement. Unintentional deposition of radioactive microspheres in tissues other than the liver may cause serious treatment complications. Therefore, a safety procedure is performed in the week(s) before the actual treatment. During this procedure, coil embolization of extrahepatic branches may be performed and a strategic catheter position is chosen before administering a (harmless) scout dose of technetium-99 m-labelled macro-aggregated 
albumin $\left({ }^{99 \mathrm{~m}} \mathrm{Tc}-\mathrm{MAA}\right)$. Afterward, SPECT/CT and planar nuclear scintigraphy are obtained to exclude the presence of extrahepatic activity and significant liver-to-lung shunting. The treatment procedure is typically performed 1-2 weeks later, with the administration of ${ }^{90} \mathrm{Y}$ microspheres from identical catheter positions, followed by posttreatment imaging with bremsstrahlung SPECT/CT or ${ }^{90}$ Y-PET/CT [24].

As a second topic of possible improvement, the intrahepatic distribution of therapeutic ${ }^{90} \mathrm{Y}$ microspheres cannot be accurately predicted in advance. The scout dose of ${ }^{99 m}$ Tc-MAA particles differs markedly in embolic effect, size, weight, and number of particles infused [25], and therefore fails to predict the intrahepatic distribution of ${ }^{90} \mathrm{Y}$ microspheres in most cases [26-28]. Besides, imaging of the ${ }^{90} \mathrm{Y}$ microspheres biodistribution itself is already a challenge due to the lack of $\gamma$-radiation emission. Traditionally, bremsstrahlung SPECT/CT has been used for posttreatment imaging, but it suffers from a low spatial resolution. Internal-pair production-based ${ }^{90} \mathrm{Y}-\mathrm{PET} / \mathrm{CT}$ has become available as an alternative for quantitative imaging, but the low count rate and inherent noise limit its applicability in daily clinical care [29-31].

Third, it is generally assumed that the preferential arterial vascularization of liver tumors will lead to a selective targeting of tumorous tissue following intra-arterial infusion of radioactive microspheres. It is known from pathological examinations of livers treated with ${ }^{90} \mathrm{Y} R E$ that radioactive microspheres cluster preferentially within the peripheral tumor vasculature. The concentration of microspheres can be up to 200 times greater in the tumor periphery than in the tumor center and the healthy liver tissue [32]. Various studies have investigated doseresponse relationships in RE. The majority of these studies found strong associations between $\mathrm{T} / \mathrm{N}$ ratios, absorbed radiation doses, tumor response and overall survival [27, 33-39]. Yet, the degree of tumor targeting, as expressed by the $\mathrm{T} / \mathrm{N}$ microsphere uptake ratio, shows marked interindividual variability in practice, with a reported range of $0.6-25.9$ [40]. This heterogeneity in $\mathrm{T} / \mathrm{N}$ uptake ratios is likely a result of various factors, including differences in tumor angiogenesis, microsphere characteristics, catheter position, and flow-bound distribution physics [25, 33]. A recent investigation demonstrated that up to $60 \%$ of patients with liver metastases treated with ${ }^{166} \mathrm{Ho}$ RE had at least one tumor that received less than or an equal amount of radioactivity as compared to the surrounding healthy liver tissue $(\mathrm{T} / \mathrm{N} \leq 1)$ [4]. Flamen et al., also reported similar findings, with $38 \%$ of the metastatic liver lesions in their study having an unfavorable $\mathrm{T} / \mathrm{N}$ uptake ratio $(<1)$ after $\mathrm{RE}$ with ${ }^{90} \mathrm{Y}$ microspheres [39]. Since unfavorable $\mathrm{T} / \mathrm{N}$ uptake ratios cannot be predicted and only become apparent after treatment, timely adjustments in treatment technique are not yet feasible.
The highly variable tumor targeting is an important clinical problem that may at least explain some of the inconsistencies in reported tumor response rates after RE $[1,41]$. Considering the reported dose-response relationship, it can be expected that improved $\mathrm{T} / \mathrm{N}$ ratios will positively affect tumor response after RE. It may also reduce hepatotoxicity, since healthy liver tissue absorbed dose has previously been correlated to biochemical toxicity [37]. Improvement of $\mathrm{T} / \mathrm{N}$ ratios is especially important in CRLM, since metastases from this tumor type are relatively hypovascular compared with other tumor types (such as neuroendocrine tumors or uvea melanoma), and generally exhibit low $\mathrm{T} / \mathrm{N}$ ratios.

The above outlined shortcomings of current RE practice are being addressed in the SIM trial, for which trial accrual has started as of November 2014. The distinctive imaging capacities and availability of an identical scout dose of ${ }^{166}$ Ho microspheres, combined with the promising effects on particle fluid dynamics facilitated by the ARC, may result in an optimized treatment technique of RE in patients with CRLM.

\section{Trial status}

Patient recruitment was ongoing at the time of submission.

\section{Additional file}

Additional file 1: Standard Protocol Items: Recommendations for Interventional Trials (SPIRIT) 2013 checklist: recommended items to address in a clinical trial protocol and related documents. (DOC $121 \mathrm{~kb}$ )

\section{Acknowledgements}

Not applicable.

Funding

Not applicable.

Availability of data and materials

Not applicable.

\section{Authors' contributions}

AvdH, JP, RB, HV, GK, ML, and MvdB were involved in the trial design, project development, and drafting of the study protocol manuscript. RB, ML, and MvdB perform the patient evaluation and treatment. GK oversees the ${ }^{166} \mathrm{Ho}$ microspheres production process. AvdH and JP collect the clinical data. AvdH will perform the statistical analysis. MvdB is the principle investigator for the trial. All authors read and approved the final manuscript.

\section{Authors' information}

Not applicable.

\section{Competing interests}

The authors declare that they have no competing interests.

\section{Consent for publication}

Not applicable.

Ethics approval and consent to participate

The SIM trial is approved by the Institutional Review Board of the University Medical Center Utrecht (reference number 14/287) and all participants will provide written informed consent to participate. 
Received: 15 June 2016 Accepted: 5 October 2016

Published online: 25 October 2016

\section{References}

1. Rosenbaum CENM, Verkooijen HM, Lam MGEH, et al. Radioembolization for treatment of salvage patients with colorectal cancer liver metastases: a systematic review. J Nucl Med. 2013;54(11):1890-5. doi:10.2967/jnumed.113.119545.

2. van den Hoven AF, Lam MGEH, Jernigan S, van den Bosch MAAJ, Buckner GD. Innovation in catheter design for intra-arterial liver cancer treatments results in favorable particle-fluid dynamics. J Exp Clin Cancer Res. 2015;34(1): 74. doi:10.1186/s13046-015-0188-8.

3. Smits MLJ, Nijsen JFW, van den Bosch MAAJ, et al. Holmium-166 radioembolisation in patients with unresectable, chemorefractory liver metastases (HEPAR trial): a phase 1, dose-escalation study. Lancet Oncol. 2012;13(10):1025-34. doi:10.1016/S1470-2045(12)70334-0.

4. Smits ML, Elschot M, van den Bosch MAAJ, et al. In vivo dosimetry based on SPECT and MR imaging of $166 \mathrm{Ho}$-microspheres for treatment of liver malignancies. J Nucl Med. 2013;54(12):2093-100. doi:10.2967/jnumed.113.119768.

5. Prince JF, van Rooij R, Bol GH, de Jong HWM, van den Bosch MJ, Lam MGEH. Safety of a scout dose preceding hepatic radioembolization with 166Ho microspheres. J Nucl Med. 2015;56(6):817-23. doi:10.2967/jnumed. 115.155564

6. Arepally A, Chomas J, Kraitchman D, Hong K. Quantification and reduction of reflux during embolotherapy using an antireflux catheter and tantalum microspheres: ex vivo analysis. J Vasc Interv Radiol. 2013;24(4):575-80. doi:10. 1016/j.jvir.2012.12.018

7. van den Hoven AF, Prince JF, Samim M, et al. Posttreatment PET-CTconfirmed intrahepatic radioembolization performed without coil embolization, by using the antireflux Surefire Infusion System. Cardiovasc Intervent Radiol. 2014;37(2):523-8. doi:10.1007/s00270-013-0674-3.

8. Fischman AM, Ward TJ, Patel RS, et al. Prospective, randomized study of coil embolization versus surefire infusion system during yttrium-90 radioembolization with resin microspheres. J Vasc Interv Radiol. 2014;25(11): 1709-16. doi:10.1016/j.jvir.2014.08.007.

9. Rose SC, Kikolski SG, Chomas JE. Downstream hepatic arterial blood pressure changes caused by deployment of the surefire antireflux expandable tip. Cardiovasc Intervent Radiol. 2013;36(5):1262-9. doi:10.1007/ s00270-012-0538-2.

10. Moher D, Hopewell S, Schulz KF, et al. CONSORT 2010 Explanation and Elaboration: Updated guidelines for reporting parallel group randomised trials. J Clin Epidemiol. 2010;63(8):e1-e37. doi:10.1016/j.jclinepi.2010.03.004.

11. Chan A-W, Tetzlaff JM, Altman DG, et al. SPIRIT 2013 statement: defining standard protocol items for clinical trials. Ann Intern Med. 2013;158(3):200-7 doi:10.7326/0003-4819-158-3-201302050-00583.

12. Eisenhauer $E$, Therasse $P$, Bogaerts J, et al. New response evaluation criteria in solid tumours: revised RECIST guideline (version 1.1). Eur J Cancer. 2009; 45(2):228-47. doi:10.1016/j.ejca.2008.10.026.

13. Siriwardena AK, Mason JM, Mullamitha S, Hancock HC, Jegatheeswaran S. Management of colorectal cancer presenting with synchronous liver metastases. Nat Rev Clin Oncol. 2014;11(8):446-59. doi:10.1038/nrclinonc.2014.90.

14. Elferink MAG, de Jong KP, Klaase JM, Siemerink EJ, de Wilt JHW. Metachronous metastases from colorectal cancer: a population-based study in North-East Netherlands. Int J Colorectal Dis. 2014;30(2):205-12. doi:10. 1007/s00384-014-2085-6.

15. Guglielmi AP, Sobrero AF. Second-line therapy for advanced colorectal cancer. Gastrointest Cancer Res. 2007;1(2):57-63.

16. Wang C-C, Li J. An update on chemotherapy of colorectal liver metastases. World J Gastroenterol. 2012;18(1):25-33. doi:10.3748/wjg.v18.i1.25.

17. Saltz LB. Second- and third-line treatment options for unresectable metastatic colorectal cancer. Gastrointest Cancer Res. 2008;2(6):299-302.

18. Cunningham D, Maroun J, Vanhoefer U, Van Cutsem E. Optimizing the use of irinotecan in colorectal cancer. Oncologist. 2001;6 Suppl 4:17-23.

19. Simkens LHJ, Koopman M, Punt CJ. Optimal duration of systemic treatment in metastatic colorectal cancer. Curr Opin Oncol. 2014;26(4):448-53. doi:10. 1097/CCO.0000000000000087.

20. Koopman M, Antonini NF, Douma J, et al. Sequential versus combination chemotherapy with capecitabine, irinotecan, and oxaliplatin in advanced colorectal cancer (CAIRO): a phase III randomised controlled trial. Lancet. 2007:370(9582):135-42. doi:10.1016/S0140-6736(07)61086-1.

21. Seymour MT, Maughan TS, Ledermann JA, et al. Different strategies of sequential and combination chemotherapy for patients with poor prognosis advanced colorectal cancer (MRC FOCUS): a randomised controlled trial. Lancet. 2007:370(9582):143-52. doi:10.1016/S01406736(07)61087-3.

22. Simkens LHJ, van Tinteren $H$, May $A$, et al. Maintenance treatment with capecitabine and bevacizumab in metastatic colorectal cancer (CAIRO3): a phase 3 randomised controlled trial of the Dutch Colorectal Cancer Group. Lancet. 2015;6736(14):1-10. doi:10.1016/S0140-6736(14)62004-3.

23. Smits MLJ, van den Hoven AF, Rosenbaum CENM, et al. Clinical and laboratory toxicity after intra-arterial radioembolization with $90 \mathrm{Y}$ microspheres for unresectable liver metastases. PLoS One. 2013;8(7):e69448.

24. Mahnken AH, Spreafico C, Maleux G, Helmberger T, Jakobs TF. Standards of practice in transarterial radioembolization. Cardiovasc Intervent Radiol. 2013; 36(3):613-22. doi:10.1007/s00270-013-0600-8.

25. Van de Wiele C, Maes A, Brugman E, et al. SIRT of liver metastases: physiological and pathophysiological considerations. Eur J Nucl Med Mol Imaging. 2012;39(10):1646-55. doi:10.1007/s00259-012-2189-6.

26. Dhabuwala A, Lamerton P, Stubbs RS. Relationship of 99mtechnetium labelled macroaggregated albumin (99mTc-MAA) uptake by colorectal liver metastases to response following Selective Internal Radiation Therapy (SIRT). BMC Nucl Med. 2005;5:7. doi:10.1186/1471-2385-5-7.

27. Ulrich G, Dudeck O, Furth C, et al. Predictive value of intratumoral 99mTcmacroaggregated albumin uptake in patients with colorectal liver metastases scheduled for radioembolization with 90Y-microspheres. J Nucl Med. 2013;54(4):516-22. doi:10.2967/jnumed.112.112508.

28. Wondergem M, Smits MLJ, Elschot M, et al. 99mTc-macroaggregated albumin poorly predicts the intrahepatic distribution of $90 \mathrm{Y}$ resin microspheres in hepatic radioembolization. J Nucl Med. 2013;54(8):1294301. doi:10.2967/jnumed.112.117614.

29. Carlier T, Eugène T, Bodet-Milin C, et al. Assessment of acquisition protocols for routine imaging of Y-90 using PET/CT. EJNMMI Res. 2013;3(1):11. doi:10. 1186/2191-219X-3-11.

30. Kao Y-H, Steinberg JD, Tay Y-S, et al. Post-radioembolization yttrium-90 PET/ CT - part 1: diagnostic reporting. EJNMMI Res. 2013;3(1):56. doi:10.1186/ 2191-219X-3-56

31. Elschot M, Vermolen BJ, Lam MGEH, de Keizer B, van den Bosch MJ, de Jong HWM. Quantitative comparison of PET and Bremsstrahlung SPECT for imaging the in vivo yttrium-90 microsphere distribution after liver radioembolization. PLoS One. 2013;8(2):e55742. doi:10.1371/journal.pone. 0055742.

32. Kennedy AS, Nutting C, Coldwell D, Gaiser J, Drachenberg C. Pathologic response and microdosimetry of (90)Y microspheres in man: review of four explanted whole livers. Int J Radiat Oncol Biol Phys. 2004;60(5):1552-63. doi: 10.1016/j.jirobp.2004.09.004.

33. Gulec S, Mesoloras G, Dezarn W, McNeillie P, Kennedy AS. Safety and efficacy of Y-90 microsphere treatment in patients with primary and metastatic liver cancer: the tumor selectivity of the treatment as a function of tumor to liver flow ratio. J Transl Med. 2007:5:15. doi:10.1186/1479-5876-5-15.

34. Lau WY, Leung WT, Ho S, et al. Treatment of inoperable hepatocellular carcinoma with intrahepatic arterial yttrium-90 microspheres: a phase I and II study. Br J Cancer. 1994;70(5):994-9.

35. Walrand S, Lhommel R, Goffette P, Van den Eynde M, Pauwels S, Jamar F. Hemoglobin level significantly impacts the tumor cell survival fraction in humans after internal radiotherapy. EJNMMI Res. 2012;2(1):20. doi:10.1186/ 2191-219X-2-20.

36. Garin $\mathrm{E}$, Lenoir $\mathrm{L}$, Rolland $\mathrm{Y}$, et al. Dosimetry based on $99 \mathrm{mTC}$ macroaggregated albumin SPECT/CT accurately predicts tumor response and survival in hepatocellular carcinoma patients treated with 90Y-loaded glass microspheres: preliminary results. J Nucl Med. 2012;53(2):255-63. doi: 10.2967/jnumed.111.094235.

37. Lam MGEH, Goris ML, lagaru AH, Mittra ES, Louie JD, Sze DY. Prognostic utility of $90 \mathrm{Y}$ radioembolization dosimetry based on fusion $99 \mathrm{mTC}$ macroaggregated albumin-99mTc-sulfur colloid SPECT. J Nucl Med. 2013; 54(12):2055-61. doi:10.2967/jnumed.113.123257.

38. Lam MGEH, Banerjee A, Goris ML, et al. Fusion dual-tracer SPECT-based hepatic dosimetry predicts outcome after radioembolization for a wide range of tumour cell types. Eur J Nucl Med Mol Imaging. 2015;2:1192-201. doi:10.1007/s00259-015-3048-z

39. Flamen $P$, Vanderlinden $B$, Delatte $P$, et al. Multimodality imaging can predict the metabolic response of unresectable colorectal liver metastases to radioembolization therapy with Yttrium-90 labeled resin microspheres. Phys Med Biol. 2008;53(22):6591-603. doi:10.1088/0031-9155/53/22/019. 
40. van den Hoven AF, Smits ML, Rosenbaum CENM, Verkooijen HM, van den Bosch MAAJ, Lam MGEH. The effect of intra-arterial angiotensin II on the hepatic tumor to non-tumor blood flow ratio for radioembolization: a systematic review. PLoS One. 2014;9(1):e86394. doi:10.1371/journal.pone. 0086394.

41. Vente MAD, Wondergem $M$, van der Tweel I, et al. Yttrium-90 microsphere radioembolization for the treatment of liver malignancies: a structured meta-analysis. Eur Radiol. 2009;19(4):951-9. doi:10.1007/s00330-008-1211-7.

Submit your next manuscript to BioMed Central and we will help you at every step:

- We accept pre-submission inquiries

- Our selector tool helps you to find the most relevant journal

- We provide round the clock customer support

- Convenient online submission

- Thorough peer review

- Inclusion in PubMed and all major indexing services

- Maximum visibility for your research

Submit your manuscript at www.biomedcentral.com/submit 